\title{
Recurrent Fever of Unknown Origin Associated with Forgotten Dental Root Infection in a Geriatric Patient \\ E Karagoz ${ }^{1}$, A Ulcay ${ }^{2}$, BT Suer ${ }^{3}$, V Turhan ${ }^{1}$, AAcar ${ }^{2}$, H Erdem $^{2}$, O Öncül ${ }^{2}$, L Görenek ${ }^{2}$
}

\begin{abstract}
Objective: To report a rare clinical condition caused by odontogenic infections; unexplained or recurrent fever related to dental abscess.

Clinical Presentation and Intervention: An 85-year-old female patient plagued with recurrent fever, lasted for more than 10 months, had been hospitalized on five occasions for diagnosis and treatment. After rigorous physical examinations, laboratory and radiological investigations, we established an infected left maxillary canine root inside her upper premaxilla, which had been forgotten during a dental procedure 10 years ago. Although there were no signs of inflammation on the panoramic radiography, the oral surgeon noted an abscess formation right beside the dental root upon extraction of the root. We cleared the inside of the upper palate and took bacterial cultures of the abscess. The sample material was directly inoculated into blood culture bottles. Granulicatella adiacens was recovered from the cultures. The patient made a full recovery subsequent to drainage of the abscess, extraction of the forgotten dental root, and antibiotic treatment.
\end{abstract}

Conclusion: Early dental evaluation and referral of patients with persistent and recurrent fever (even without any oral symptoms) to a dentist has a critical role to prevent unnecessary, timeconsuming further diagnostic tests and invasive procedures. A simple panoramic radiography may be useful to establish the diagnosis.

Keywords: Dental infection, fever of unknown origin, Granulicatella adiacens

From: ${ }^{1}$ Department of Infectious Diseases and Clinical Microbiology, Van Training and Research Hospital, Van, ${ }^{2}$ Department of Infectious Diseases and Clinical Microbiology, GATA Haydarpasa Training Hospital, Istanbul, and ${ }^{3}$ Department of Oral Surgery, GATA Haydarpasa Training Hospital, Turkey.

Correspondence: Dr E Karagoz, Department of Infectious Diseases and Clinical Microbiology Van Training and Research Hospital, Van, Turkey. E-mail: ergenekonkaragoz@hotmail.com 


\section{INTRODUCTION}

Fever of unknown origin (FUO) is a common diagnostic problem and may arise as a consequence of rare infections, neoplasms and connective tissue diseases. An 85-year-old female patient plagued with recurrent fever, which had lasted for more than 10 months, had been hospitalized on five occasions for diagnosis and treatment. After rigorous physical examinations, laboratory and radiological investigations, we established an infected dental root inside her upper palate, which had been forgotten during a dental procedure 10 years ago. This procedure rendered her edentulous, thus at the time of these admissions she had no tooth. Granulicatella adiacens was identified in cultures of the dental root abscess. An endodontic infection was found to be the cause of fever in this case. The patient made a full recovery subsequent to drainage of the abscess, extraction of the forgotten dental root, and antibiotic treatment.

\section{CASE PRESENTATION}

An 85-year-old female patient was admitted to the emergency department due to persistent and recurrent fever with rigor and chills as well as general weakness lasting for 10 months. She seemed well despite her fever of $39.5^{\circ} \mathrm{C}$. Her vital signs and systemic examination were normal. On her first visit, the patient was hospitalized and followed-up in the Infectious Diseases Department. Her initial white blood cell (WBC) level was 25,000/mm ${ }^{3}$. Following investigations, she was diagnosed with chronic cystitis and urinary tract infection (UTI) caused by extended spectrum beta lactamase (ESBL) positive Escherichia. coli as detected by urine culture. One gram Ertapenem once daily for 7 days was administered, after which she was discharged. The patient visited our clinic exactly 5 days later with high fever and was hospitalized for further work-up. 
Her urinalysis revealed leukocyturia. Her additional labwork revealed: WBC:

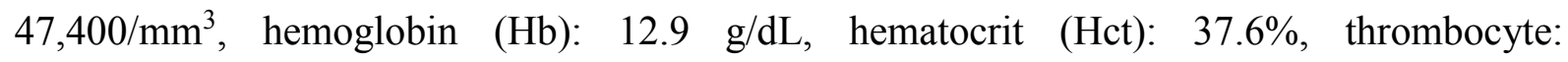
$388,000 / \mathrm{mm}^{3}$, erythrocyte sedimentation rate: $90 \mathrm{~mm} /$ hour, C-reactive protein (CRP): 133 $\mathrm{mg} / \mathrm{L}$, aspartate aminotransferase (AST): $32 \mathrm{U} / \mathrm{L}$, alanine transaminase (ALT): $30 \mathrm{U} / \mathrm{L}$, blood urea nitrogen (BUN):29 mg/dL, and creatinine: $1.16 \mathrm{mg} / \mathrm{dL}$. Imipenem at a dose of $500 \mathrm{mg} 4$ times daily was commenced. No abnormalities were observed on her chest radiography and organomegaly was ruled out on abdominal ultrasonography (USG). However, on abdominal computed tomography (CT), a splenic artery aneurysm and 3 aspects of diverticulosis in the duodenum and sigmoid colon were detected.

Cardiovascular surgery consultation to ascertain if the splenic artery aneurysm, which was thought to be a mycotic aneurysm, was the cause of the fever suggested otherwise and surgery was not recommended. In urinary USG, radiological findings that accompanied chronic cystitis were detected. We continued antibiotherapy for 14 days and the patient was discharged again. She was hospitalized 3 more times and the primary cause of her fever could not be detected in our investigations. Interestingly, her fever and leukocyte count normalized one day and three days respectively after the initiation of antibiotherapy on each occasion of her admission.

On the sixth visit, the patient was hospitalized due to the same complaints and was investigated in a detailed manner with a number of laboratory and radiological tests, including biochemical tests, abdominal CT, colonoscopy and panoramic radiography. On this visit, her lab values were as follows: WBC: $21,500 / \mathrm{mm}^{3}$, erythrocyte sedimentation rate: $23 \mathrm{~mm} / \mathrm{hour}$, CRP: $15 \mathrm{mg} / \mathrm{L}$. Her blood and urine cultures were found to be negative. Interestingly, she had no urinary symptoms, which was different from prior visits. Additional radiological examinations, such as X ray, USG of the abdominal cavity, cystoscopy and transthoracic echocardiography were performed and no pathological changes were discovered. 
Supplementary examinations of the digestive tract were also performed. On abdominal CT and colonoscopy several diverticula were noticed. Although the patient was edentulous and did not complain of tooth related pain, in light of our knowledge about her dental procedure years ago, the patient referred to the department of oral surgery in the same institution for further oral examination.

During her dental radiographic examination, oral surgeon noticed the impacted left maxillary canine root, which probably had been left during previous dental extraction of canine tooth (Figure). Although there were no signs of inflammation on the panoramic radiography, the oral surgeon noted an abscess formation right beside the dental root upon the surgical extraction of the root. Oral surgeon took the root fragment out and cleaned the inside of the socket and took cultures of the abscess. The sample material was directly inoculated into blood culture bottles. Granulicatella adiacens was recovered from the cultures. We administered amoxicillin and clavulanic acid 1 gram perorally for 7 days and discharged our patient. Our patient's fever has not repeated for 9 months. Physical examination and laboratory findings were normal in her follow-up visits.

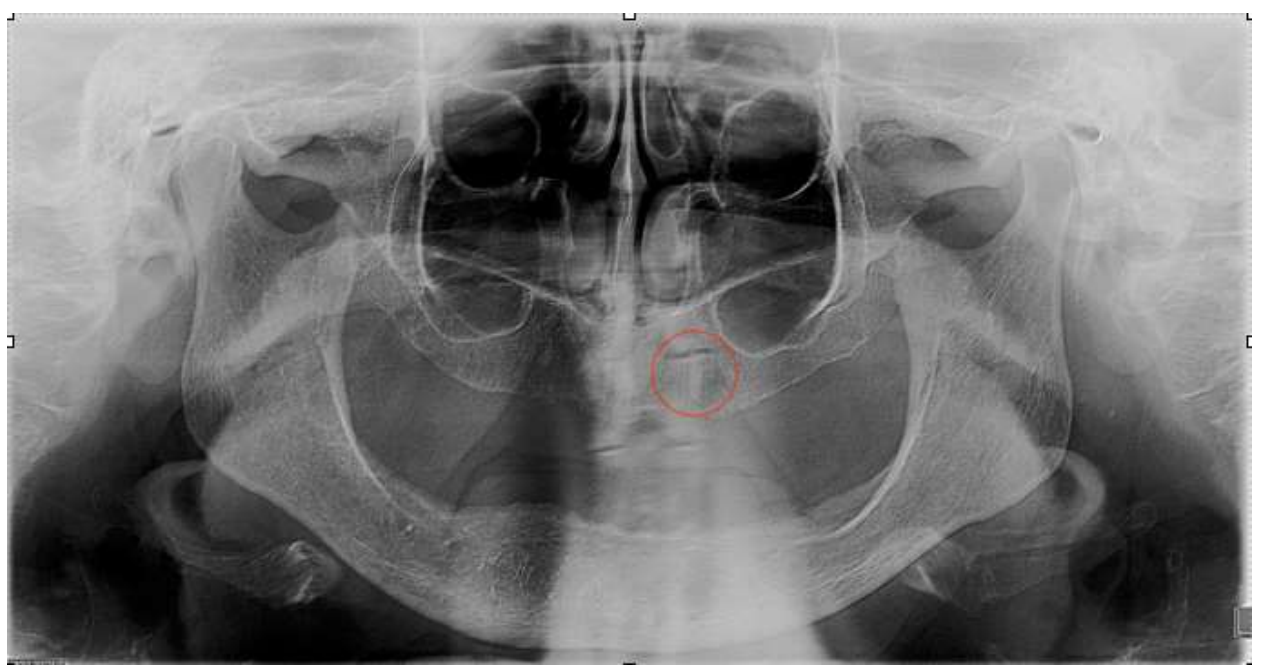

Figure: Red circle indicates the root fragment of upper maxillary left canine. 


\section{DISCUSSION}

Since FUO is a diagnostic hurdle, several investigations should be performed to make an appropriate diagnosis. According to Arnow and Flaherty (1), as well as Hague(2), at least the following examinations should be performed in relation to a recognized case of FUO: a comprehensive history, repeated physical examination, complete blood count (CBC), erythrocyte sedimentation rate (ESR), urea and electrolytes, liver function tests, antinuclear antibodies, rheumatoid factor, routine blood cultures, urine analysis and urine culture, faecal occult blood and stool culture, tuberculin skin test (Mantoux test), HIV antibodies, cytomegalovirus IgM antibodies, a CT of the abdomen and a chest X-ray (1). Among the most and widely known causes of FUO are infections.

Despite being described in the textbooks of the dentistry as periapical or periodontal infections, they are not regarded as frequent causes of FUO. Furthermore, books of internal medicine do not highlight oral pathology while evaluating a patient presenting fever (3). Consequently, in the process of the differential diagnosis of FUO, doctors seldom take into account dental infections. In the variety of cases, patients have acute pain along with local symptoms or there are some apparent finding concerning the diagnosis. Still, the cases that evade early detection present non specific or non localized signs, and thus, they go undetected (3).

In our patient, a number of investigations were undertaken - blood cell count, liver enzyme levels, urea and electrolytes, an X-ray of the chest, electrocardiography, ultrasonography and computer tomography of the abdominal cavity and also colonoscopy. Yet, we could not detect any pathological etiology determining the condition of the patient. Although she had no teeth in her mouth and she was not suffering from toothache, we performed a pantomogram since all her teeth had been removed by a dental operation 10 years ago. 
On pantomogram, we detected a dental root. Though there was no inflammatory aspect in the radiogram, when we extracted the root, we detected an abscess formation beside the dental root. Granulicatella spp. are innately found in oral flora. Dental abscess and endodontic infection can be caused by the referred microorganism $(3,4)$. Furthermore, Granulicatella spp. can lead to a variety of serious infections $(2,4,5)$. Some major examples of these infections include infective endocarditis, bloodstream infections, septic arthritis, mycotic aneurysms and brain abscesses $(2,4)$. In the case of endocarditis, Granulicatella spp. arise as a culture negative endocarditis since this microorganism bears a slow-growing characteristic. Therefore, it is thought to be an uncommon cause of infection. In another trial conducted by Rocas IN et al, the novel oral species leading to endodontic infections were investigated using PCR methods with samples obtained from symptomatic cases (6). The most frequently detected microorganisms were Dialister invisus, Olsenna uli and Granulicatella adiacens. It is inflexible to assert that these bacteria are always found in symptomatic infections (7).

It is very difficult to cultivate these microorganisms by classical conventional culture methods, therefore a PCR method can facilitate the detection of responsible microorganisms. However, we opportunely managed to detect the pathogenic microorganism by culture methods. Culture samples that were taken from abscess material and inoculated into two blood culture bottles did grow and we detected Granulicatella adiacens. In our case, symptoms disappeared after treatment involving dental extraction and a course of antibiotics. In conclusion, dental infections may be responsible for FUO. Early diagnosis and treatment is crucial to prevent undesirable and life-threatening complications such as infective endocarditis. Therefore, dental examination should never be underestimated or neglected in patients with FUO. 


\section{REFERENCES}

1. Arnow PM, Flaherty JP. Fever of unknown origin. Lancet 1997; 350: 575-80.

2. Hague R. Managing the child with a fever. Practitioner. 2015; 259: 17-21.

3. Karachaliou IG, Karachalios GN, Kanakis KV, Petrogiannopoulos CL, Zacharof AK. Fever of unknown origin due to dental infections: cases report and review. Am J Med Sci 2007; 333: 109-10.

4. Cargill JS, Scott KS, Gascoyne-Binzi D et al. Granulicatella infection: diagnosis and management. J Med Microbiol 2012; 61: 755-61.

5. Hsiao WW, Li KL, Liu Z,et al. Microbial transformation from normal oral microbiota to acute endodontic infections. BMC genomics 2012; 13: 345.

6. Rocas IN, Siqueira JF, Jr. Detection of novel oral species and phylotypes in symptomatic endodontic infections including abscesses. FEMS Microbiol letters. 2005; 250: 279-85.

7. Arslan F, Karagöz E, Arslan BY, Mert A. An unnoticed origin of fever: periapical t tooth abscess. Three case reports and literature review. Infez Med. 2016; 24: 67-70. 